\title{
El Programa de Expansión y Mejoramiento de la Educación Rural en Entre Ríos, Argentina (1978-1991)1
}

\author{
Eva Mara Petitti² \\ ORCID: 0000-0001-8014-7181
}

\section{Resumen}

El artículo estudia el Programa de Expansión y Mejoramiento de la Educación Rural que se llevó a cabo durante los años '80 en todas las provincias argentinas menos la de Buenos Aires, financiado por el Banco Interamericano de Desarrollo. Incluía un sistema de nuclearización de escuelas, acompañado de políticas de diagnóstico, perfeccionamiento docente, regionalización del currículo, dotación de equipamiento y refacciones, ampliaciones y construcciones de edificios escolares. El objetivo de este trabajo es abordar el Programa como un objeto de estudio en sí mismo que permita desarrollar una agenda de investigación que vincule diferentes problemas. En primer lugar, el proceso de regionalización y transferencia de las escuelas primarias. En segundo término, las modificaciones en el diseño institucional y el personal a cargo del programa. En tercer lugar, las diferentes apropiaciones del EMER por parte de los diversos actores. Finalmente, las distancias entre la propuesta del Programa y las realidades locales. La investigación se centra en la provincia de Entre Ríos y se construye en base a una abundante documentación escrita que se encuentra en archivos de la provincia y a partir de entrevistas realizadas a diferentes actores que participaron del Programa. En las conclusiones, el trabajo pretende articular las cuatro problemáticas presentadas.

\section{Palabras clave}

Programa EMER - Educación rural - Entre Ríos - Argentina

1- Agradezco a Susana Meyer el haber llamado mi atención sobre las posibilidades de profundizar acerca de la temática.

2- Universidad Nacional de Entre Ríos CONICET- Instituto de Estudios Sociales, Entre Ríos, Argentina. Contacto: mpetitti@fceco.uner.edu.ar. 


\section{The Program for the Expansion and Improvement of Rural Education in Entre Ríos, Argentina (1978-1991)}

\section{Abstract}

The article studies the Program for the Expansion and Improvement of Rural Education that took place during the '80s in all the Argentine provinces except Buenos Aires, funded by the Inter-American Development Bank. It included a system of nuclearization of schools, accompanied by diagnostic policies. The main objectives of the project are to improve the quality of teaching, regionalization of the curriculum, provision of equipment and spare parts, as well as expansion and construction of school buildings. The objective of this work is to address the Program as an object of study in itself in order to develop a research agenda linking different problems. Firstly, the process of regionalization and transfer of primary schools. Secondly, the changes in the institutional design and the staff in charge of the program. Thirdly, the different appropriations of EMER by the different actors of Member State. Finally, the gaps between the Program proposal and the local realities. The research focuses on the province of Entre Rios and will be built drawing from abundant documentation found in archives of the province and based on interviews with different actors who participated in the Program Project. For the sake of conclusion, the study aims to articulate the four problems presented.

\section{Keywords}

EMER Program - Rural education - Entre Rios - Argentina.

\section{Introducción}

El Programa EMER, cuya sigla corresponde a Expansión y Mejoramiento de la Educación Rural, estuvo destinado a todas las provincias argentinas menos la de Buenos Aires (ENTRE RÍOS, 1980b). Incluía un sistema de nuclearización de escuelas, acompañado de políticas de diagnóstico, perfeccionamiento docente, regionalización del currículo, dotación de equipamiento y refacciones, ampliaciones y construcciones de edificios escolares. En el caso de Entre Ríos se aplicó a cuatro departamentos ubicados en el norte de la provincia -Feliciano, Federal, La Paz y Federación-, aquellos que poseían los mayores índices de ruralidad, analfabetismo y deserción escolar (PETITTI; SCHMUCK; CHELLOTTI, 2018). Financiado por el Banco Interamericano de Desarrollo (BID) y la nación en partes iguales, tuvo como objetivo lograr "[...] un mejor rendimiento del sistema educativo" entendiendo por tal el primario y “[...] una ampliación de los servicios educacionales en el ámbito rural” (ENTRE RÍOS, 1980b, p. 11) concibiendo por ámbito 
rural todo aquel agrupamiento poblacional inferior a 2.000 habitantes (ENTRE RÍOS, 1980b). Más allá de las dificultades para establecer una periodización exacta, debido a las etapas y subprogramas que lo conformaron y los gobiernos de diferente signo político, -autoritarios y democráticos- que atravesó, ubicamos el Programa entre 1978 y 1991.

A pesar de la señalada complejidad que revista, la enorme cantidad de material que se ha producido en ese marco y el numeroso personal involucrado en su ejecución, son escasas las investigaciones que han profundizado en este Programa. El hecho de que se trate de un proyecto iniciado durante el gobierno dictatorial (1976-1983) y desarrollado a lo largo de la década del '80, puede haber influido en el poco interés por abordarlo. En las entrevistas realizadas a hombres y mujeres que formaron parte del EMER, se evidencia una intención de diferenciar sus propias trayectorias profesionales del contexto dictatorial en el surgió el proyecto. Como muestra Laura Rodríguez, en plena democracia las autoridades educativas de 1988 anunciaban que se estaba "elaborando un proyecto de Regionalización, Descentralización y Nuclearización Educativa destacando el carácter democratizador del mismo y evitando mencionar la experiencia descentralizadora que se llevó a cabo durante la dictadura" (RODRÍGUEZ, 2010, p. 458).

La existencia del EMER ha sido mencionada en algunas investigaciones generales sobre programas educativos (CAILLODS; JACINTO, 2006). Entre aquellos que se han acercado al tema, podemos citar para la provincia de Salta el trabajo de Marcelo Jorge Navarro (2014), quien destina un capítulo de su tesis al retorno a la democracia con el análisis del Programa. Por su parte Nora Yentel (2011) en un estudio de caso sobre la Escuela Media Experimental Técnico Agropecuaria creada en el marco del Programa de Expansión y Mejoramiento de la Educación Técnica Agropecuaria (en adelante EMETA), da cuenta de lo sucedido en Neuquén con el EMER. Para Entre Ríos, encontramos algunas referencias en el trabajo de Susana Mayer (2016).

En líneas generales, el Programa EMER ha sido estudiado como un antecedente directo del Programa EMETA. ${ }^{3}$ Uno de los primeros trabajos al respecto es el de Elisa Cragnolino (2000), quien analiza el caso de Córdoba y arroja luz sobre las limitaciones del proyecto modernizador y los condicionamientos institucionales y estructurales. Más recientemente, Lorena Leguizamón observa para la provincia de La Rioja una “[...] constante búsqueda de generación de recursos propios puesto que la entrega de los fondos no se correspondía con los tiempos de implementación de la propuesta" (LEGUIZAMÓN, 2014, p. 14). En cuanto a su aplicación a nivel provincial, Talía Gutiérrez señala la existencia de "[...] disparidades entre lo planteado en los convenios y las realidades diversas de la educación agraria, pero también dificultades relacionadas con el ineficiente manejo de los fondos, o rivalidades políticas" (GUTIÉRREZ, 2012, p. 6). Más adelante, Talía Gutiérrez, Jorge Navarro y Celeste de Marco sostienen que la mayoría de las escuelas funcionaron en regiones estratégicas y recibieron un gran impulso inicial, pero que la aplicación del Plan EMETA “[...] fue dispar en las distintas provincias” (GUTIÉRREZ; NAVARRO; DE MARCO, 2014, p. 12). En el caso de Salta, Navarro y Gutiérrez evidencian que las escuelas

3- Es posible, por lo tanto, que el Programa EMETA haya sido más estudiado que el EMER porque su aplicación coincidió con el regreso del gobierno democrático. 
EMETA “[...] siguieron funcionando a pesar del desfinanciamiento de la educación técnica, y lograron sostenerse en el tiempo, siendo ejes del desarrollo local en cada una de sus comunidades, siendo hasta el día de hoy centros educativos de referencias" (NAVARRO; GUTIÉRREZ, 2013, p. 116). Todos estos trabajos, si bien forman parte de investigaciones más amplias, constituyen un importante aporte que debemos considerar a la hora de estudiar el EMER.

En este artículo nos interesa abordar el Programa como un objeto de estudio en sí mismo que permita desarrollar una agenda de investigación que vincule diferentes problemas. Para ello estamos revisando numerosos documentos que se elaboraron en el marco del Programa ubicados en distintos archivos y centros de documentación de la provincia de Entre Ríos. ${ }^{4}$ Por otra parte realizamos entrevistas a quienes fueron durante esos años maestros, instructores de talleres, directores de escuelas, jefes de departamentos y personal a cargo del programa, así como a un conductor encargado del traslado de los alumnos. En este trabajo, luego de presentar sintéticamente las características generales del programa, propondremos una agenda de investigación con cuatro posibles temas a desarrollar: el financiamiento internacional de la educación en el marco del proceso de regionalización y transferencia de las escuelas primarias; las modificaciones en el diseño institucional y el personal que conformó las distintas reparticiones del Estado que se encargaron de un mismo programa que atravesó diferentes gobiernos; las tensiones vinculadas a la aplicación y la apropiación de las políticas educativas que se dieron entre un programa financiado por el BID y su implementación en las escuelas; y finalmente, la distancia entre el diseño del Programa y las realidades locales.

\section{Una aproximación al programa en EMER en Entre Ríos}

Si observamos el programa en el ámbito nacional, encontramos que no todas las provincias se incorporaron al mismo tiempo. El EMER fue estructurado por el Ministerio de Educación de la Nación en apoyo y complemento de la acción educativa de las provincias en el nivel primario. Según la Memoria del Consejo Federal de Educación (1976-1983) en una primera etapa, participaron diecisiete provincias, en una segunda fueron incorporadas Mendoza, La Pampa y Córdoba y finalmente se agregó Santa Fe (ARGENTINA, 1983).

En 1978 se creó en Entre Ríos una comisión dependiente de la Secretaría de Estado de Cultura y Educación para la elaboración de los estudios de perfectibilidad y factibilidad. En un plazo muy breve, el documento final fue evaluado por el BID, la Nación y la Provincia (ENTRE RÍOS, 1978). El 24 de marzo de 1979 se aprobó el acuerdo firmado entre el Estado nacional y el BID para poner en ejecución el Programa EMER (ENTRE RÍOS, 1979). Ese mismo día, se sancionó el decreto 1175 por el cual se aprobó un préstamo del BID de 66 millones de dólares destinado a la ejecución de un Programa Global de Ciencia y Tecnología compuesto por dos subprogramas, uno a ejecutar por el Instituto Nacional de Ciencia y Técnica Hídrica y el otro por el Consejo Nacional de Investigaciones Científicas y Técnicas (ALAGAÑARAZ; BEKERMAN, 2014).

4- Nos referimos al archivo del Consejo General de Educación, el Centro de Información Educativa del Consejo General de Educación, el Archivo Histórico y el Archivo Administrativo de la Provincia de Entre Ríos, así como a los archivos de las Escuelas 14 de Feliciano, 48 de Federal y 50 de La Paz. 
La coordinación y administración del Programa a nivel nacional quedó a cargo de una Unidad Ejecutora Central (UEC) bajo dependencia del Ministerio de Cultura y Educación. A su vez las provincias debían crear Unidad Ejecutoras Provinciales (UEP) y firmar un convenio con el Ministerio de Cultura y Educación.

El monto del financiamiento ascendía a los 100 millones de dólares, de los cuales la mitad era aportada por la nación y la otra mitad por el BID, mediante un préstamo amortizable en 25 años de plazo con un interés del 3\% anual sobre saldos (ARGENTINA, 1983). El préstamo no debía ser reintegrado por las provincias, sin embargo, como contraparte requería de la estructura y el personal provincial.

Según el convenio, para participar de la ejecución del Programa, las provincias debían elevar una propuesta que la UEC debía evaluar. Una vez aprobada la propuesta, la nación transfería los recursos a las provincias, cuya suma en Entre Ríos ascendía a seis millones de dólares. Se destinaron principalmente a obras civiles y en menor medida para cubrir la compra de equipos y materiales y para llevar a cabo cursos de perfeccionamiento docente. Si bien hay muchos aspectos vinculados al plan -especialmente años de funcionamiento y escuelas involucradas-, en los cuales los entrevistados plantean diferencias, existe consenso a la hora de identificar la cantidad de recursos que estipuló el programa (ENTRE RÍOS, 1980a).

Un aspecto a destacar es que abarcó gobiernos de diferente signo político, tanto dictatoriales con las intervenciones de Carlos Enrique Aguirre (1978-1981), Jorge Washington Ferreyra (1981-1983) y Mario César Bertozzi (1983), como democráticos con la gestión radical de Sergio Montiel (1983-1987) y el gobierno peronista de Jorge Busti (1987-1991). El EMER se compuso por un conjunto de cinco subprogramas, cada uno de ellos con un responsable a cargo: 1) Elaboración del currículum (además de los contenidos mínimos y uniformes para todo el país, se debían explicitar los contenidos de carácter regional, a lo cual se sumaban los talleres); 2) Capacitación docente; 3) Construcción de escuelas (nuevas o refacción y ampliación de las existentes); 4) Equipamiento de las escuelas (mobiliario, equipo y materiales); y 5) Desarrollo de la comunidad (promoción de la salud y la extensión cultural).

A lo largo del periodo mencionado el Programa constó de diferentes etapas: 1) Previamente a su ejecución cuando se realizaron estudios de factibilidad (1978-1981); 2) le siguió la primera etapa de implementación donde se realizaron acciones de capacitación docente, investigaciones y la regionalización del currículo y se llevaron a cabo las obras de edificación y se equipó a las escuelas (1981-1986); 3) luego se desarrolló la segunda etapa, cuando se dio comienzo al sistema nuclearizado durante 1986; 4) Finalmente, se creó una Unidad de Seguimiento y Evaluación que se extendió durante los últimos años de la implementación del sistema nuclearizado y que finalizó en 1991.

La existencia de diferentes subprogramas, las distintas etapas que transitaron y los diferentes gobiernos que atravesó el Programa, hacen difícil establecer una periodización. Todas las preguntas que realizamos acerca del inicio y la finalización del Programa fueron seguidas de un silencio, al que le continuó la manifestación de una duda y una respuesta hipotética, que se extiende según los testimonios desde 1978 a 1986 en lo que respecta a su inicio y desde 1990 a 1998 en relación a su finalización. 
Una vez suscripto el contrato de préstamo, en 1980 se firmó el convenio con el Ministerio de Cultura y Educación de la Nación y a partir de entonces se creó la UEP del EMER en Entre Ríos que estaría facultada para administrar y disponer de fondos -manejar una cuenta especial donde se depositaría el financiamiento-, adquirir bienes y coordinar acciones con otras reparticiones de la provincia (el Consejo General de Educación, Ministerio de Acción Social, Ministerio de Obras y Servicios Públicos) (ENTRE RÍOS, 1980a). Las UEP fueron, durante los ‘90, “[...] las terminales locales de la articulación vertical” que los organismos internacionales adoptaron para llevar adelante la ejecución de sus programas y proyectos." Junto a las UEC obraron como "una forma de garantizar el correcto uso de los recursos asignados.” (BARRETO; ZAVALA, 2004, p. 111).

La UEP era un ente descentralizado de la administración pública provincial con jerarquía de Subsecretaria de Estado, dependiente del Ministerio de Hacienda, Economía, Obras y Servicios Públicos. Estaba a cargo de un coordinador y su estructura integrada por un asesor legal y cuatro áreas: Infraestructura y Equipamiento, Administrativo-Contable, Seguimiento y Evaluación y el Programa Educativo (ENTRE RÍOS, 1980a). El equipo, que se conformó entre 1981 y 1982, estaba integrado por veintitrés miembros, contaba con un coordinador, un asesor, cuatro directores, técnicos y auxiliares. El área Programa Educativo quedó conformada por los sectores de Investigación, Perfeccionamiento Docente, Currículo y Promoción de la Comunidad, para cuyas direcciones convocaron a especialistas de diferentes disciplinas. Entre 1981 y 1984 se llevaron a cabo acciones en el marco de los distintos subprogramas y a partir de 1983 se realizaron obras de refacción, ampliación y edificación de establecimientos educativos incluidos en el plan y se dotaron a las escuelas núcleo de equipamiento (ENTRE RÍOS, 1984).

El sistema de nuclearización consistió en veinte conjuntos de escuelas integrados por tres o seis escuelas satélites que trasladaban a sus alumnos de sexto y séptimo grado una o dos veces por semana a las escuelas núcleo, donde luego de las actividades curriculares almorzaban y realizaban talleres. Estos formaban parte del área de orientación laboral (consistían en cursos electricidad, soldadura o carpintería para los varones y artes del hogar -corte y confección y cocina- para las mujeres), área agropecuaria (huerta, jardín, pequeño vivero y cría de pequeños animales) y un área artístico-expresiva (música y educación física). Los directores de escuelas núcleo se convertían en intermediarios entre el supervisor y los directores de las escuelas satélites. A la vez, éstos últimos debían colaborar con la escuela núcleo y organizar en sus propias escuelas huertas con asesoramiento de los instructores de actividades agropecuarias.

En 1986 se comenzaron a realizar pruebas en siete de los veinte conjuntos nuclearizados incluidos en el programa y en 1987 se aprobó la resolución que adoptó el sistema. Los traslados de los niños se realizaban en camionetas destinadas al programa y cada una estaba a cargo de un chofer, cuyo rol en el Programa era central y aún hoy así se lo recuerda.

Como entre las condiciones formales para la recepción del préstamo y la puesta en marcha del programa, el BID estipulaba una cantidad de recursos por alumno y condiciones de factibilidad relacionadas con la ubicación de las escuelas y el traslado, no todos los establecimientos fueron incorporados al Programa. Los veinte conjuntos nuclearizados 
abarcaban 114 escuelas satélites de las 210 existentes en los departamentos incluidos en el programa (ENTRE RÍOS, 1987). ${ }^{5}$

El inicio de la nuclearización, en 1986 coincidió con la finalización del financiamiento del Programa. A partir de entonces dejó de depender de la Unidad Ejecutora Provincial y pasó a estar a cargo del Consejo General de Educación. ${ }^{6}$ En 1987 fue absorbido por la UEP del Programa EMETA y al año siguiente se creó una Comisión Integradora para la Educación Agrotécnica (CIPEA) que tendría a su cargo la conducción de los Programas EMER y EMETA, con participación de la Coordinación Ejecutiva Programas EMER-EMETA, el Consejo General de Educación y la secretaría ministerial de asuntos agrarios (ENTRE RÍOS, 1988a). Dependientes de la Coordinación Ejecutiva funcionaban la UEP Programa EMETA y la Unidad de Seguimiento Evaluación y Expansión Programa EMER que se mantuvo hasta 1991, cuando su personal fue trasladado al Consejo General de Educación (ENTRE RÍOS, 1988b). No obstante ello, el acercamiento a los archivos escolares da cuenta de que a pesar de que el programa dejó de funcionar a nivel estatal, la impronta de la nuclearización continuó en algunos establecimientos y se recuperó para hacer traslados de alumnos a medida que se fue ampliando la edad de obligatoriedad escolar.

\section{Cuatro notas para la construcción de una agenda de investigación}

Uno de los primeros aspectos para profundizar en relación a este Programa consiste en el financiamiento internacional de la educación en el marco del proceso de transferencia de las escuelas primarias de la nación a las provincias que finalizó en 1978 (RUIZ; MAUCERI, 2010; RODRIGUEZ, 2008). Teniendo en cuenta que la creación de la comisión para la elaboración de estudios de perfectibilidad y factibilidad del Programa EMER coincide con la sanción del decreto que aprobaba la transferencia, cabe preguntarse en qué medida el Plan EMER, que según sus fundamentos tenía como fin "[...] un mejor rendimiento del sistema educativo[...]" y “[...] una ampliación de los servicios educacionales en el ámbito rural[...]” (ARGENTINA, 1983, p. 29), fue una forma de que la nación, a través de endeudamiento externo, contribuyera a atenuar las consecuencias económicas de la transferencia. Un argumento en este sentido, lo constituyen las declaraciones del ministro de Cultura y Educación, doctor Rafael Llerena Amadeo, quien afirmaba que el convenio que daba origen al Programa EMER “[...] demuestra que nuestro sistema educativo no se ha resquebrajado al transferir las escuelas primarias nacionales a jurisdicción provinciales.” (LA NACIÓN, 1979, p. 3). Asimismo, en la Memoria del Consejo Federal de Cultura y Educación para el período 1976-1983 consta que "El Programa EMER ha sido estructurado por el Ministerio de Educación de la Nación en apoyo y complementación de la acción educativa de Nivel Primario que realizan las provincias en el ámbito rural" (ARGENTINA, 1983, p. 31).

\footnotetext{
5- De esas 114 escuelas participaban 6.256 alumnos, una población escolar de 6.798 niños, 400 docentes y 6.741 hogares.

6- Si bien hay documentos escritos y testimonios que dan cuenta que en 1986 el Programa pasó al Consejo General de Educación, el decreto de transferencia se sancionó al año siguiente. Véase Decreto 1399, 13 de marzo de 1987.
} 
El BID al igual que el Banco Mundial (BM) cobra mayor presencia en las políticas educativas, tanto en Argentina como en los demás países de América Latina durante la década de los 90. Hasta entonces la Organización de las Naciones Unidas para la Educación, la Ciencia y la Cultura (UNESCO) fue el organismo más influyente en materia de producción de conocimiento sobre la problemática educativa, mientras que el BID ha promovido y financiado actividades de investigación relacionadas con el análisis de las políticas educativas (GALARZA; SUASNÁBAR; MERODO, 2007; ASCOLANI, 2008; CAUSA, 2011). Ahora bien, ¿cómo se explica por lo tanto el financiamiento del Programa EMER por el BID a fines de los años '70? ¿Es posible que la vinculación se pueda establecer por el énfasis en el desarrollo rural contenido en el Programa o por los aspectos de investigación y evaluación educativa que contenía?

Nora Krawczyk señala que para los organismos internacionales la reforma con más trascendencia en la región "[...] fue la operación de un nuevo modelo de organización y gestión del sistema y de la escuela, por medio de la descentralización y de la autonomía escolar." Con estas acciones, continúa Krawczyk, "[...] se pretendía reducir los gastos del gobierno central en educación.” (KRAWCZYK, 2002, p. 634). La descentralización política y administrativa formó parte del discurso de los países centrales y de los organismos internacionales que operaban en Latinoamérica (RODRÍGUEZ, 2008). Como muestra Ascolani (2008) para el caso del BM, los préstamos en Argentina fueron asignados para respaldar transformaciones profundas del sistema educativo, como la descentralización educativa en los años '90.

Si bien cuando observamos Entre Ríos -que en líneas generales fue uno de los estados provinciales con menor porcentaje de escuelas nacionales- encontramos que en los departamentos donde se desarrolló el EMER las escuelas nacionales tuvieron escasa presencia, es cierto que, como señala Tedesco (1983), la transferencia fue justificada tanto en términos político-administrativos como en términos curriculares. En el caso de Argentina, la descentralización aparece como un mecanismo de adecuación a las posibilidades de cada sector social y región. En esa línea Romina de Luca (2013) da cuenta de la correspondencia entre la descentralización y la regionalización del currículum. A lo largo del gobierno militar se elaboraron diagnósticos por parte de la nación sobre la marcha de la regionalización y todas las medidas adoptadas se encaminaron a garantizar una injerencia mayor de las realidades locales sobre el currículum. ¿Consistió entonces, en una forma de introducir la regionalización de la educación que ha estado vinculada a la transferencia? Es posible suponer, teniendo en cuenta la dificultad que a lo largo del siglo XX encontró la posibilidad de establecer contenidos diferenciados para los ámbitos urbanos y rurales, que la regionalización del currículum se pretendió introducir a partir de políticas vinculadas a la educación rural que en la década del 80 tuvieron una fuerte vinculación con el Programa EMER.

En el caso de Brasil, el Banco Mundial llevó a cabo el proyecto EDU RURAL durante los años '80 vinculado a sus estrategias de reducir la pobreza en países latinoamericanos focalizando en préstamos en la educación primaria a las regiones más desfavorecidas, destinado a construcción de escuelas, insumos y capacitación (ASCOLANI, 2008). Es decir, los mismos aspectos que los priorizados por el BID en Argentina en el marco del EMER. 
Asimismo, en Brasil esa década estuvo marcada por un proceso de nuclearización de escuelas rurales que condujo al cierre de las escuelas aisladas y unidocentes (DE SOUZA, 2013). Diferente fue el programa EMER donde la nuclearización no condujo al cierre de las escuelas rurales, ya que el traslado se restringía solo a los alumnos de sexto y séptimo grado, uno o dos días a la semana. Sin embargo, al igual que en el EMER, el proceso de nuclearización se hizo en torno a comunidades rurales centrales en relación a las demás y el transporte escolar ocupó un lugar central, dejando fuera del proceso a aquellas localidades sin comunicación (DE SOUZA, 2013). Laura Rodríguez (2008) muestra que, en la provincia de Buenos Aires, la única que no fue afectada al EMER, la política de transferencia fue diferente en las zonas urbanas y en las rurales ya que en estas últimas se crearon proyectos de concentración -que consistía en la fusión en un solo edificio de varias escuelas- que promovieron el cierre de cientos de establecimientos para zonas rurales. Pero también las políticas destinadas a las escuelas rurales fueron variando y se pasó de un proyecto de cierre a uno de nuclearización.

Por otra parte, la elección de establecimientos en las áreas rurales para la implementación del tercer ciclo de EGB, desde la sanción de la Ley Federal de Educación en 1993, la incorporación de la secundaria completa con la Ley Nacional de Educación de 2006 y la modalidad de doble jornada Escuelas Nina en Entre Ríos, a partir de una resolución provincial de 2012, se basó en la mayoría de los casos en aquellos establecimientos que con anterioridad habían sido escuelas núcleo. A partir de ese dato también es posible preguntarnos en qué medida el programa sirvió de base para la implementación de otras políticas educativas que se llevaron a cabo posteriormente.

El segundo aspecto que nos interesa profundizar se relaciona con el diseño institucional y los funcionarios y técnicos que conformaron las distintas reparticiones del Estado que se encargaron de un programa que atravesó diferentes gobiernos. Siguiendo a Germán Soprano y Ernesto Bohoslavsky pretendemos abordar el Programa EMER desde una perspectiva que analice al Estado "desde adentro", como un "espacio polifónico en el que se relacionan y se expresan grupos” (BOHOSLAVSKY; SOPRANO, 2010, p. 24). Desde esa óptica, al abordar un programa que atravesó diferentes gobiernos y que estuvo a cargo de diferentes reparticiones, cabe preguntarse acerca de los permanentes acomodamientos al interior del Estado y la interlocución con otros actores estatales y no estatales.

Se trata de un programa educativo que, como decíamos más arriba, hasta 1986 estuvo a cargo de una UEP, repartición externa al Consejo General de Educación, y que al año siguiente pasó a depender de la UEP del Programa EMETA en carácter de remanente. ${ }^{7}$ Teniendo en cuenta esas modificaciones, retomamos de Soprano y Bohoslavsky (2010) el interrogante acerca de quiénes fueron, cómo se legitimaron y contra quienes tuvieron que competir, en este caso, los encargados de diseñar y llevar a cabo el programa EMER en la provincia de Entre Ríos a lo largo de los diferentes gobiernos que se sucedieron entre 1978 y 1991. ¿Cuáles fueron los factores que influyeron en las modificaciones de la localización estatal del Programa? ¿Existieron superposiciones de funciones entre la UEP y el Consejo General de Educación? ¿En qué medida esas modificaciones, así como las alianzas y tensiones al interior de la propia repartición y con aquellas que

7- El 15 de noviembre de 1984 por decreto 4421 se había creado la UEP del Proyecto EMETA. 
estaba estrechamente vinculada, influyeron en la toma de decisiones? ¿Cuáles fueron las trayectorias y experiencias de los integrantes del programa a medida que cambiaron las gestiones de gobierno y el programa sufrió modificaciones en su localización estatal?

Daniel Lvovich en su análisis sobre el reclutamiento de funcionarios municipales durante la dictadura propone buscar la explicación del consenso social en las regularidades de la vida burocrática mucho más que en el nivel de la ideología. Por un lado "[...] pensar en márgenes de acción menos estrechos que los habitualmente supuestos, y no lógicas no necesariamente heterónomas que orientaron sus prácticas" (LVOVICH, 2010, p. 425). En segundo lugar, considerar a los protagonistas como "[...] burócratas que encontraron en la dictadura la posibilidad de un ascenso no por fidelidad sino por su eficacia y por la eliminación de competidores para ocupar esos cargos” (p. 427). En tercer lugar, suponer que "[...] las regularidades de la vida burocrática o las pequeñas ambiciones personales incidieron sobre la decisión de asumir cargos públicos en un contexto de dictadura posibilitando así el funcionamiento efectivo de las instituciones estatales de distinta importancia y nivel” (p. 428). Finalmente, “[...] pensar en los usos estratégicos de las limitadas posibilidades de participación que abrió el régimen militar” (p. 428). Consideramos que en estos señalamientos podemos encontrar algunas claves para abordar nuestro objeto de estudio.

Por otra parte, la participación mayoritaria de mujeres en el área educativa del Programa EMER nos conduce a una relectura de los trabajos de Laura Rodríguez (2010) sobre su participación en cargos burocráticos durante la dictadura. En el periodo de conformación del grupo de trabajo, el sector vinculado a educación que se agregó a UEP del Programa -que se constituyó sobre la base de la UEP destinada al financiamiento del Programa de Salto Grande- estaba conformado exclusivamente por mujeres. Si bien dependían jerárquicamente del coordinador de la UEP, que era hombre y militar, el programa se asentó sobre esta área. Siguiendo los trabajos de Lvovich y Rodríguez es posible sostener que las responsables del Área Educativa aceptaron trabajar en el programa "[...] atendiendo a sus propios intereses y motivaciones de ascender en la carrera profesional” (RODRÍGUEZ, 2010, p. 458).

Con el retorno al gobierno democrático, con la excepción del coordinador y la responsable del Área Educativa, sus responsables continuaron en el programa. En 1986 pasaron de la UEP que funcionaba en un edificio propio a un grupo de oficinas, cuyo número se fue reduciendo con el tiempo, en el Consejo General de Educación. El vínculo personal entre las encargadas del Área Educativa y el personal del Consejo, fue tenso y las integrantes del Programa entrevistadas manifestaron no solo sentirse hostigadas por las nuevas autoridades sino también haber tenido que luchar para mantenerlo en marcha. En 1987 fueron trasladadas a otra oficina, como parte de una pequeña sección del EMER que quedó dependiente del Programa EMETA y continuaron allí hasta que finalizó. Luego, algunas de ellas regresaron al Consejo y trabajaron principalmente en programas con financiamiento.

Como decíamos al inicio, los diferentes actores entrevistados manifestaron disimiles respuestas ante la pregunta sobre y el inicio y la finalización del programa. Elisa Cragnolino llamó nuestra atención al respecto y nos incentivó a seguir profundizando en esta cuestión. Por lo tanto, el tercer aspecto que nos interesa abordar son las tensiones 
vinculadas a la reformulación y la apropiación de las políticas educativas provenientes del programa EMER por parte de los actores involucrados. Para ello tendremos en cuenta las tramas de relaciones de poder entre los principales actores implicados: responsables del programa, jefes de departamentos, supervisores, directores de escuelas núcleos y satélites, conductores encargados de hacer el traslado, maestros, instructores de talleres, alumnos y familias. Hasta donde hemos indagado, los puntos de tensión más importante tuvieron que ver con las responsabilidades y funciones de cada uno, el uso compartido del equipamiento, el traslado de los alumnos y el cumplimiento de los cursos de perfeccionamiento. Así, algunos directores de escuelas satélites se resistieron a que sus alumnos se subieran a una camioneta y fueran trasladados por miedo a que tuvieran un accidente y no hubiera un seguro que se hiciera responsable, como también otros se negaron a aceptar que alumnos de escuelas satélites utilizaran los recursos didácticos del propio.

De esta forma, poniendo el foco en el EMER, nos proponemos estudiar las políticas educativas, distinguiendo en cada escala -internacional, nacional, regional, local- el desarrollo de procesos con determinada profundidad (ACHILLI, 2000, 2013; ROCKWELL, 2009). Más precisamente, nos preguntamos en qué medida estas acciones de orden institucional se mediatizan en las prácticas de los funcionarios provinciales y los actores vinculados al espacio social rural (CRAGNOLINO, 2004).

Como hemos visto en la nota anterior los rostros humanos del Estado intervienen en la mediación. Las encargadas de área educativa llegaban a las escuelas no solo con el mandato recibido. Profesionales de las Ciencias de la Educación, trabajadoras sociales, psicólogas fueron a las escuelas con su conocimiento técnico y con sus expectativas respecto al programa. Laura Rodríguez señala que "[...] no existió una correspondencia lineal y unívoca entre el hecho de ocupar un cargo en el gobierno autoritario y de hacer literalmente lo que solicitaran el ministro o gobernador" (RODRÍGUEZ, 2010, p. 458), en este caso el coordinador de la UEP. Las encargadas de dictar los cursos de perfeccionamiento docente, de diseñar el currículum, de establecer vínculos con la comunidad y de investigar, tuvieron un margen de acción importante en relación a los lineamientos del BID que financió el programa. Al igual que con el préstamo que el BID otorgó a Conicet en 1979, si bien el gobierno, en este caso provincial, "[...] tuvo que responder a condiciones formales para la recepción del préstamo y puesta en marcha del programa, no detectamos la existentica de condicionamientos" (ALGAÑARAZ; BEKERMAN, 2014, p. 15) en el diseño del sistema nuclearizado ni en el desarrollo de cada uno de los subprogramas. Esas decisiones fueron tomadas por el personal de área educativa de la UEP, integrada por educadoras, psicólogas y asistentes sociales, entre otras, y muchas de sus acciones trascendieron lo meramente establecido en la normativa. Así, las encargadas del área educativa en la UEP de Entre Ríos, manifiestan en las entrevistas realizadas, que dictaron cursos que no estuvieron incluidos en el programa, publicaron resultados de investigaciones cuyas cifras hablaban de las limitaciones de los resultados de la política educativa hasta el momento, hicieron participar de las reuniones con docentes y directivos, a los jefes municipales y a los curas, e instaron a que las mujeres no solo asistieran a los de talleres de labores del hogar sino que también concurrieran a los de carpintería. ${ }^{8}$

$\mathbf{8}$ - Entrevistas realizadas por la autora, los días 10 de noviembre de 2017, 27 de noviembre 2017, 20 de febrero de 2018, 10 de marzo de 2018, 21 de abril de 2018, Paraná, Ente Ríos. 
Pero además, entre las encargadas del programa y los alumnos, mediaron las maestras, los conductores encargados de hacer los traslados, los instructores de los talleres, los directores de escuelas núcleo y satélites y los supervisores que dependian de la Dirección General de Escuelas, todos ellos con expectativas diferentes tanto en el plano personal como respecto al programa. Así como muchos fueron críticos con la obligación de realizar los perfeccionamientos los días sábados, otros aprovecharon el puntaje que otorgaban los cursos para ascender en el escalafón y llegaron a supervisores y jefes de departamento. Asimismo, un aspecto central del programa fue el transporte escolar intracampo. Uno de los conductores del programa a quien hemos entrevistado relata que como no había ambulancia, usaban la camioneta si había que llevar enfermos a la ciudad más cercana. Por otra parte "[...] se los llevaba [a los niños] a fin de año, se hacía con las cooperadoras, los llevábamos a Concordia, pasábamos a Uruguay, a Santa Fe, el túnel [subfluvial Uranga-Begnis], esos chicos de Las Achiras no conocían ni Cimarrón y acá se integraban, pagaban el combustible" (Entrevista, 13/4/18, Entre Ríos).

El último aspecto a mencionar consiste en la distancia entre el diseño del Programa y las realidades locales. Estimamos que podemos allanar el camino a la respuesta de por qué el Programa no dio resultado a largo plazo en lo cual coinciden los diferentes actores entrevistados.9 Las responsables del Área Educativa del Programa, enfatizan que las dificultades para que diera resultados residieron en la falta de compromiso por parte de las familias de los niños, y destacan importantes diferencias entre uno de los departamentos -Federación- y el resto, cuyos índices de analfabetismo y ruralidad eran mayores a los de éste. Asimismo, señalan que parte de la responsabilidad recayó en los docentes que preparaban a los niños para que una vez que terminaran la escuela pudieran irse a la ciudad. Uno de los directivos entrevistados relata que los responsables de las escuelas satélites no se tomaban en serio las capacitaciones (Entrevista, 12/4/18, Entre Ríos).

Consideramos que una respuesta a la dificultad de que el plan diera sus resultados tiene que ver con que no tuvo en cuenta los problemas estructurales del territorio. Uno de los choferes del Programa señalaba "A las escuelas del campo le hicieron mucha reforma el EMER, les llevaban heladera, lamentablemente le llevaban heladera eléctrica y no había luz" (Entrevista, 13/4/18, Entre Ríos).

También relativo a la electricidad, un ex maestro de una escuela satélite del departamento de Federal recordaba ante la pregunta sobre si los chicos aplicaban los conocimientos que recibían en los talleres: "El sistema de huerta sí, pero electricidad, en este caso no porque no teníamos como" (Entrevista, 12/4/18, Entre Ríos). En este mismo sentido, un ex instructor de los talleres manifestaba que "[...] lo que enseñaba en la Encierra [una de las escuelas núcleo] era más productivo, porque se enseñaba carpintería y acá [otra de las escuelas núcleo] se enseñaba un circuito, pero no tenían electricidad" (Entrevista, 12/4/18, Entre Ríos).

Vinculado con lo anterior, sostenemos como hipótesis que la dificultad de que diera resultados en el largo plazo se debió a que el Programa no consideró el espacio social ni a

9- Para muchos de los actores, mientras funcionó fue bueno para los niños, pero una vez que se terminaron los recursos el programa llegó a su fin. Rescatan como resultados del programa las refacciones y las construcciones de edificios escolares, así como los equipos, aunque estos últimos se fueron perdiendo cuando finalizaron los talleres por la falta de mantenimiento. 
los actores rurales, sino que más bien los colocó en un lugar pasivo. En las investigaciones llevadas a cabo en el marco del EMER se deja de manifiesto que las familias no se ocupaban de la educación de sus hijos. Por ejemplo, en las conclusiones de un estudio publicado en 1983 se delimitaron ciertas características de los cuatro departamentos del norte y se manifestó que:

Las familias no tienen una clara idea respecto de la finalidad de la educación primaria y la misión de la escuela. En la mayoría de los casos, la escuela es un lugar al que "hay que ir" por algún tiempo. En el mejor de los casos, es vista como una instancia que hay que atravesar para que el chico aprenda a "leer y sacar cuentas" y a "comportarse como la gente". [...] La incomprensión llana respecto de la función de la escuela trae aparejada una necesaria ausencia de expectativas respecto de la finalidad de la educación para el futuro de los niños y la comunidad. (ENTRE RÍOS, 1983, p. 2).

Eso contrasta con los resultados de investigaciones académicas realizadas en los últimos años que dan cuenta del accionar de las familias en la creación y construcción de escuelas. En su trabajo sobre la educación rural en Santa Fe entre 1916 y 1932, Adrián Ascolani (2012) muestra el interés de muchos agricultores por la educación de sus hijos. Asimismo Lucía Lionetti (2010) y Elisa Cragnolino (2004, 2011) han evidenciado el lugar de las familias en las creaciones de las escuelas rurales y el control de los docentes. De hecho, las escuelas 14 de Feliciano y 69 de La Paz que fueron incluidas en el plan, contaron con la participación activa de las familias en la creación de los establecimientos y la construcción de sus edificios.

Cabe dejar planteado algún interrogante sobre las causas que explican las diferencia entre lo propuesto por el Estado y el accionar de las comunidades rurales en la construcción de escuelas, es decir, estudiar no solo las representaciones que las comunidades reproducen en torno al Estado sino también las representaciones que el Estado reproduce en torno a las comunidades.

\section{Notas finales}

El trabajo se centró en la provincia de Entre Ríos, pero consideramos que es fundamental seguir avanzando en el desarrollo del Programa EMER en otras provincias. Lo que se sabe para los casos de Neuquén (YENTEL, 2011) y Salta (NAVARR0, 2014) y lo que estamos comenzando a indagar incorporando la provincia de Córdoba (CRAGNOLINO; PETITTI; SCHMUCK, 2018) es indicativo de que en cada provincia tuvo un desenvolvimiento diferente.

A lo largo de este artículo de carácter exploratorio, son más los interrogantes que las certezas que hemos presentado. Los discursos de los actores entrevistados que han ocupado diferentes roles en el programa están tejidos por una periodización propia, que no siempre se condice con las fechas de los decretos y resoluciones que se sancionaron en el marco del programa, sino que más bien están supeditados a la propia participación, así como a las tensiones profesionales, de género y partidarias. Podemos concluir que en relación 
a los cuatro posibles abordajes que hemos planteado, hay un eje transversal. Se trata de la posibilidad de profundizar en cómo las periodizaciones de la historia política, atadas a los cambios de gobierno, se vuelven más complejas cuando abordamos las políticas educativas. Tal vez, a medida que vayamos encontrando respuestas a los interrogantes planteados, consigamos comprender por qué, a pesar de su complejidad como objeto de estudio, el análisis del Programa EMER quedó relegado en un segundo plano.

\section{Referencias}

ACHILLI, Elena. Hacer antropología social: Ios desafíos del análisis a distintas escalas. Boletín de Antropología y Educación, Buenos Aires, v. 6, n. 9, p. 103-107, 2013.

ALGAÑARAZ, Víctor; BEKERMAN, Fabiana. El préstamo BID-CONICET: un caso de dependencia financiera en la política científica de la dictadura militar argentina (1976-1983). In: BEIGEL, Fernanda; SABEA, Hanann (Coord.). Dependencia académica y profesionalización en el sur: perspectivas desde la periferia. Río de Janeiro: Ediunc: Sephis, 2014. p. 129-140.

ARGENTINA. Consejo Federal de Cultura y Educación. Secretaría Permanente. Memoria: años 1976-1983, Buenos Aires, oct. 1983.

ASCOLANI, Adrián. Escuela primaria rural en Argentina: expansión, orientación y dificultades (1916-1932). Revista Teias, Rio de Janeiro, v. 28, n. 14, p. 309-324, 2012.

ASCOLANI, Adrián. Estrategias del Banco Mundial para el financiamiento de la educación en los países latinoamericano. Educação, Porto Alegre, v. 31, n. 2, p. 139-156, maio/ago. 2008.

BARRETO, Miguel; ZAVALA, José. Articulación vertical de la inversión urbana en el nordeste argentino: reflexiones sobre la experiencia del Banco Mundial durante la década del '90. Revista de Estudios Urbanos Regionales, Santiago de Chile, v. 30, n. 90, p. 103-119, 2004.

BOHOSLAVSKY, Ernesto; SOPRANO, Germán (Ed.). Un Estado con rostro humano: funcionarios e instituciones estatales en Argentina (desde 1880 a la actualidad). Buenos Aires: Prometeo, 2010.

CAILLODS, Françoise; JACINTO, Claudia. Mejorar la equidad de la educación básica: lecciones de programas recientes en América Latina. Paris: Instituto Internacional de Planeamiento de la Educación, 2006.

CAUSA, Matías. Organismos internacionales y campo educativo: ideas político-pedagógicas entre 1976 y 1989. Páginas de Educación, Montevideo, v. 4, n. 1, p. 139-159, 2011.

CRAGNOLINO, Elisa. El acceso a la educación básica rural en familias campesinas de Córdoba (Argentina), como resultado de múltiples historias. Em aberto, Brasilia, DF, v. 24, n. 85. p. 145-162, 2011.

CRAGNOLINO, Elisa. Escuelas, maestros y familias en el espacio social rural tulumbano. Revista Etnia, Olavarría, n. 46-47, p. 1-18, 2004. 
CRAGNOLINO, Elisa. La modernización de la escuela agrotécnica. Cuadernos de Educación, Córdoba, v. 1, n. 1, p. 179-190, 2000.

CRAGNOLINO, Elisa; PETITTI, Mara; SCHMUCK, Emilia. Apuntes sobre periodizaciones y escalas a propósito del análisis de procesos... In: SEMINARIO-TALLER. RED DE INVESTIGACIÓN EN ANTROPOLOGÍA Y EDUCACIÓN (RIAE), 4., 2018, Córdoba. Anais... Córdoba: Universidad Nacional de Córdoba, Córdoba, 2018. p. 17.

DE LUCA, Romina. La educación argentina en épocas de la última dictadura militar: regionalización y descentralización del nivel primario de educación (1976-1983). Contextos Educativos, La Rioja, n. 16, p. 73-88, 2013.

DE SOUZA, María Antonia. La educación del campo en la experiencia colectiva de los movimientos sociales: indagando sobre la educación rural en Brasil. In: LIONETTI, Lucía; CIVERA, Alicia; CORREA WERLE, Flavia (Comp.). Sujetos, comunidades rurales y culturas escolares en América Latina. Rosario: Prohistoria, 2013. p. 163-178.

ENTRE RÍOS. Ministerio de Economía Obras y Servicios Públicos. Decreto 1163, 24 de marzo de 1979. Entre Ríos: Archivo del Consejo General de Educación de Entre Ríos, 1979.

ENTRE RÍOS. Ministerio de Economía Obras y Servicios Públicos. Decreto 7102, 30 de diciembre de 1988. Entre Ríos: Archivo del Consejo General de Educación de Entre Ríos, 1988b.

ENTRE RÍOS. Secretaría de Estado de Cultura y Educación. Decreto número 1485, 5 de mayo de 1978. Entre Ríos: Archivo del Consejo General de Educación, 1978.

ENTRE RÍOS. Secretaría de Estado de Cultura y Educación. Decreto número 4691, 31 de diciembre de 1980. Entre Ríos: Archivo del Consejo General de Educación, 1980a.

ENTRE RIOS. Unidad Ejecutora Provincial. Reglamento para la ejecución del Programa EMER: anexo al Convenio Nación-Provincia de Entre Ríos. Entre Ríos: Archivo del Consejo General de Educación, 1980b.

ENTRE RÍOS. Unidad Ejecutora Provincial. Programa EMER. Informe de Investigación, 1983. Entre Ríos: Archivo del Consejo General de Educación de Entre Ríos, 1983.

ENTRE RÍOS. Unidad Ejecutora Provincial. Programa EMER. Puesta en marcha de la modalidad nuclearizada. Entre Ríos: Centro de Documentación e Información Educativa del Consejo General de Educación de Entre Ríos, 1987.

ENTRE RÍOS. Unidad Ejecutora Provincial. Programa EMER. Seguimiento y Evaluación de la Modalidad Nuclearizada. Folleto 990. Entre Ríos: Centro de Documentación e Información Educativa del Consejo General de Educación de Entre Ríos, 1988a.

ENTRE RÍOS. Unidad Ejecutora Provincial. Programa EMER. Subprogramas 1984. Folleto 989. Entre Ríos: Centro de Documentación e Información Educativa del Consejo General de Educación de Entre Ríos, 1984. 
GALARZA, Daniel; SUASNÁBAR, Claudio; MERODO, Alicia. Los organismos intergubernamentales e internacionales. In: PALAMIDESSI, Mariano; SUASNÁBAR, Claudio; GALARZA, Daniel (Ed.). Educación, conocimiento y política. Buenos Aires: Manantial, 2007. p. 135-148.

GUTIÉRREZ, Talía. Políticas educativas y enseñanza agraria, una relación compleja. Buenos Aires (Argentina), 1960-2010. Revista Iberoamericana de Educación, Madrid, v. 58, n. 3. p. 1-14, 2012.

GUTIÉRREZ, Talía; NAVARRO, Jorge; DE MARCO Celeste. Educación agraria del centenario al bicentenario, 1910-2010. Bernal: Universidad Nacional de Quilmes, 2014.

KRAWCZYK, Nora. La reforma educativa en América Latina desde la perspectiva de los organismos multilaterales. Revista Mexicana de Investigación Educativa, Ciudad de México, v. 7, n. 16, p. 626-63, 2002.

LA NACIÓN, Buenos Aires, p. 3, 1979. "Para mejorar la enseñanza rural se firmó un convenio". Miércoles 5 de septiembre de 1979.

LEGUIZAMÓN, Lorena. Educar trabajando y produciendo: la aplicación del programa de expansión y mejoramiento de la educación técnico-agropecuaria en el noroeste argentino. Revista Ciencias Pedagógicas e Innovación, Santa Elena, v. 2, n. 2, p. 6-15, dic. 2014.

LIONETTI, Lucía. Las escuelas de primeras letras en el escenario social de la campaña bonaerense (18501875). Naveg@mérica, Murcia, n. 4, p. 1-21, 2010.

LVOVICH, Daniel. Burócratas, amigos, ideólogos y vecinalistas: el reclutamiento de funcionarios municipales de Morón durante la Dictadura Militar (1976-1983). In: BOHOSLAVSKY, Ernesto; SOPRANO, Germán (Ed.). Un Estado con rostro humano: funcionarios e instituciones estatales en Argentina (desde 1880 a la actualidad). Buenos Aires: Prometeo, 2010. p. 411-430.

MAYER Susana. Escuelas secundarias rurales: estudio descriptivo de la modalidad rural de educación secundaria en Entre Ríos. Entre Ríos: Universidad Autónoma de Entre Ríos, 2016. Informe Final. Proyectos de Investigación de Desarrollo Anual y Plurianual. Facultad de Ciencia y Tecnología, CS UADER 246/14. 2016.

NAVARRO Jorge. Estado y educación rural en el noroeste argentino: diversidad cultural y desigualdad educativa desde mediados del siglo XX al presente. 2014. 265 p. Tesis (Doctorado en Ciencias Sociales y Humanas) - Universidad Nacional de Quilmes (UNQ), Buenos Aires, 2014.

NAVARRO Jorge; GUTIÉRREZ, Talía. Experiencias de educación técnica rural en escuelas públicas del norte Argentino: el caso de Salta. Educação em Foco, Belo Horizonte, v. 16, n. 22, p. 99-118, 2013.

NAVARRO, Jorge; SÁNCHEZ, Lorena. Prácticas docentes rurales en contextos de globalización. Revista del Cisen, Salta, v. 2, n. 2, p. 59-80, 2014.

PETITTI; Mara; SCHMUCK, Emilia; CHELOTTI, Teresa. Políticas en territorios rurales: la educación en el norte de Entre Ríos. EJES de Economía y Sociedad, Entre Rios, v. 2, n. 3, p. 69-93, 2018. 
ROCKWELL, Elsie. La experiencia etnográfica: historia y cultura en los procesos educativos. Buenos Aires: Paidós. 2009.

RODRÍGUEZ, Laura. Gobierno municipal, descentralización educativa y funcionarios en la Provincia de Buenos Aires durante la última dictadura. In: BOHOSLAVSKY, Ernesto; SOPRANO, Germán (Ed.). Un estado con rostro humano: funcionarios e instituciones estatales en Argentina (desde 1880 a la actualidad). Buenos Aires: Prometeo, 2010. p. 431-462.

RODRÍGUEZ, Laura. La primaria durante la dictadura: reforma curricular y concentración de escuelas rurales. Archivos de Ciencias de la Educación, La Plata, v. 2, n. 2, p. 58-102, 2008.

RUIZ, Guillermo; MAUCERI, María Laura. La finalización de la educación primaria nacional: Ios últimos años de la acción directa del estado nacional en la educación primaria. Período 1961-1978. Buenos Aires: Sociedad Argentina de Historia de la Educación, 2010. p. 123-147.

TEDESCO, Juan Carlos. Elementos para una sociología del currículum escolar en Argentina. In: TEDESCO, Juan Carlos; BRASLAVSKY, Cecilia; CARCIOFI, Raúl. El proyecto educativo autoritario: Argentina (19761982). Buenos Aires: Flacso, 1983. p. 17-74.

YENTEL, Nora. Cambio en educación: acerca de los aspectos que concurren a la configuración de una trama de interrupción: un estudio de caso. 2011. 425 p. Tesina (Maestría en Ciencias de la Educación) Facultad de Filosofía y Letras, Universidad de Buenos Aires, Buenos Aires, 2011.

Recibido en: 30.04.2018 Aprobado en: 25.09.2018

Eva Mara Petitti es doctora en Historia por la Universidad Nacional de Mar del Plata. Investigadora asistente del Conicet, trabaja en el Instituto de Estudios Sociales (CONICETUNER). Jefe de trabajos prácticos en la Facultad de Ciencias Económicas de la Universidad Nacional de Entre Ríos. 